IJPSR (2011), Vol. 2, Issue 1 (Review Article)
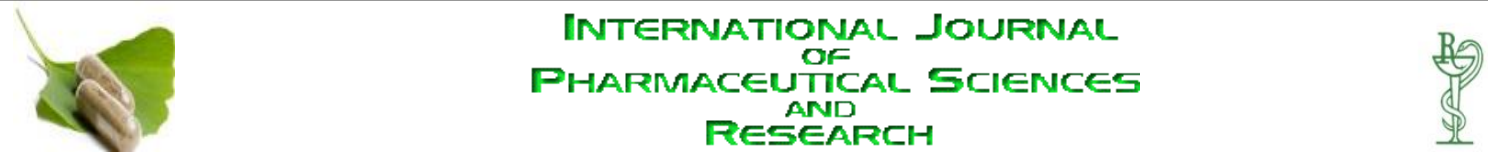

Received on 18 August, 2010; received in revised form 17 November, 2010; accepted 25 December, 2010

\title{
BUCCAL CONTROL DRUG DELIVERY SYSTEM: A REVIEW
}

Shivam Tayal* and Nishiprakash Jain

Sagar Institute of Research and Technology-Pharmacy, Ayodhya Bypass Road, Bhopal, (M.P.), India

\begin{abstract}
Keywords:

Buccal controlled drug delivery, Buccal mucosa, Buccal cavity, Dynamics, Permeation enhancers

Correspondence to Author:

Shivam Tayal

Sagar Institute of Research and Technology-Pharmacy, Ayodhya Bypass Road, Bhopal, (M.P.), India

Buccal controlled drug delivery system has been developed since the environment of the oral cavity provides potential sites for drug delivery. The acid hydrolysis and first pass effects can be avoided. The release of drug can be affected by continuous secretion of saliva. The mucin film exists in oral mucosa offers an opportunity to develop mucoadhesive system, which retain at absorption site for prolonged time by mucoadhesive binding. The close contact with absorption membrane causes more absorption of the drug. The $\mathrm{pH}$ of buccal cavity ranges between 5-7, and does not cause any problem to the drug with the right dosage form design and formulation; the permeability and the local environment of the mucosa can be controlled and manipulated in order to accommodate drug permeation. Buccal drug delivery is a promising area for continued research with the aim of systemic delivery of orally inefficient drugs as well as a feasible and attractive alternative for non-invasive delivery of potent peptide and protein drug molecules. The objective of this article is to review mucosa as a route for drug delivery by discussing the structure, environment, permeability, dynamics etc. of the buccal mucosa and the materials used for oral permeation enhancers.
\end{abstract}




\section{INTRODUCTION:}

\section{Buccal mucosa}

Structure: The total area of the oral cavity is about $100 \mathrm{~cm}^{2}{ }^{1}$. Out of this about one third is the buccal surface, which is lined with an epithelium of about $0.5 \mathrm{~mm}$ thickness (Fig. 1). The keratinized and non keratinized regions of the oral epithelium differ from each other in terms of lipid composition of the cells. The keratinized epithelium has predominantly neutral lipids (e.g., ceramides) while the non keratinized epithelium has few but polar lipids, particularly cholesterol sulphate and glucosylceramides ${ }^{2}$.

Buccal membrane has numerous elastic fibers in the dermis, which is another barrier for diffusion of drug across the buccal membrane. Drug that penetrates this membrane enters the systemic circulation via network of capillaries and arteries. The lymphatic drainage almost runs parallel to the venous vascularization and ends up in the jugular ducts. The oral mucosal surface is constantly washed by the saliva (daily turn out is about 0.5 to 2 liters). The drug absorption across the oral mucosa occurs in the non-keratinized sections for protein/peptide delivery buccal route offers distinct benefits over other mucosal routes like nasal, vaginal, rectal, etc.

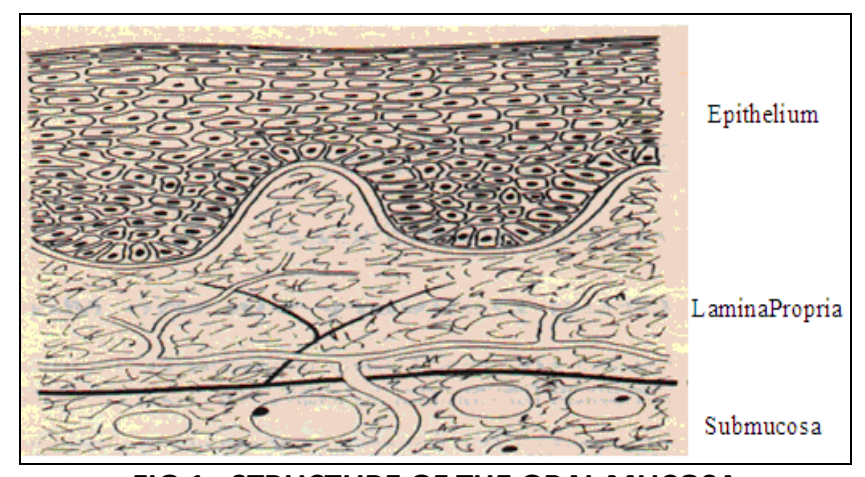

FIG 1: STRUCTURE OF THE ORAL MUCOSA

Permeability: The oral mucosa in general is somewhat leaky epithelia intermediate between that of the epidermis and intestinal mucosa. It is estimated that the permeability of the buccal mucosa is 4-4000 times greater than that of the skin ${ }^{3}$. As indicative by the wide range in this reported value, there are considerable differences in permeability between different regions of the oral cavity because of the diverse structures and functions of the different oral mucosa. In general, the permeability's of the oral mucosa decrease in the order of sublingual greater than buccal and buccal greater than palatal ${ }^{4}$. This rank order is based on the relative thickness and degree of keratinization of these tissues, with the sublingual mucosa being relatively thin and on-keratinized, the buccal thicker and non-keratinized, and the palatal intermediate in thickness but keratinized.

It is currently believed that the permeability barrier in the oral mucosa is a result of intercellular material derived from the so-called 'membrane coating granules' (MCG) ${ }^{5}$. When cells go through differentiation, MCGs start forming and at the apical cell surfaces they fuse with the plasma membrane and their contents are discharged into the intercellular spaces at the upper one third of the epithelium. This barrier exists in the outermost $200 \mu \mathrm{m}$ of the superficial layer. Permeation studies have been performed using a number of very large molecular weight tracers, such as horseradish peroxides ${ }^{6}$ and lanthanum nitrate ${ }^{7}$.

When applied to the outer surface of the epithelium, these tracers penetrate only through outermost layer or two of cells. When applied to the sub mucosal surface, they permeate up to, but not into, the outermost cell layers of the epithelium. According to these results, it seems apparent that flattened surface cell layers present the main barrier to permeation, while the more isodiametric cell layers are relatively permeable. In both keratinized and non-keratinized epithelia, the limit of penetration coincided with the level where the MCGs could be seen adjacent to the superficial plasma membranes of the epithelial cells. Since the 
same result was obtained in both keratinized and non-keratinized epithelia, keratinization by itself is not expected to play a significant role in the barrier function ${ }^{6}$. The components of the MCGs in keratinized and non-keratinized epithelia are different, however ${ }^{8}$.

The MCGs of keratinized epithelium are composed of lamellar lipid stacks, whereas the nonkeratinized epithelium contains MCGs that are nonlamellar. The MCG lipids of keratinized epithelia include sphingomyelin, glucosylceramides, ceramides, and other nonpolar lipids, however for non-keratinized epithelia, the major MCG lipid components are cholesterol esters, cholesterol, and glycosphingolipids ${ }^{8}$. Aside from the MCGs, the basement membrane may present some resistance to permeation as well, however the outer epithelium is still considered to be the rate limiting step to mucosal penetration. The structure of the basement membrane is not dense enough to exclude even relatively large molecules.

Environment: The cells of the oral epithelia are surrounded by an intercellular ground substance, mucus, the principle components of which are complexes made up of proteins and carbohydrates. These complexes may be free of association or some maybe attached to certain regions on the cell surfaces. This matrix may actually play a role in cellcell adhesion, as well as acting as a lubricant, allowing cells to move relative to one another ${ }^{9}$. Along the same lines, the mucus is also believed to play a role in bioadhesion of mucoadhesive drug delivery systems ${ }^{10}$. In stratified squamous epithelia found elsewhere in the body, mucus is synthesized by specialized mucus secreting cells like the goblet cells, however in the oral mucosa; mucus is secreted by the major and minor salivary glands as part of saliva ${ }^{9,11}$. Up to $70 \%$ of the total mucin found in saliva is contributed by the minor salivary gland ${ }^{9,11}$. At physiological $\mathrm{pH}$ the mucus network carries a negative charge (due to the sialic acid and sulfate residues) which may play a role in mucoadhesion. At this $\mathrm{pH}$ mucus can form a strongly cohesive gel structure that will bind to the epithelial cell surface as a gelatinous layer ${ }^{12}$. Another feature of the environment of the oral cavity is the presence of saliva produced by the salivary glands. Saliva is the protective fluid for all tissues of the oral cavity. It protects the soft tissues from abrasion by rough materials and from chemicals. It allows for the continuous mineralization of the tooth enamel after eruption and helps in re-mineralization of the enamel in the early stages of dental caries ${ }^{13}$. Saliva is an aqueous fluid with $1 \%$ organic and inorganic materials.

The major determinant of the salivary composition is the flow rate which in turn depends upon three factors: the time of day, the type of stimulus, and the degree of stimulation ${ }^{9,11}$. The salivary $\mathrm{pH}$ ranges from 5.5 to 7 depending on the flow rate. At high flow rates, the sodium and bicarbonate concentrations increase leading to an increase in the $\mathrm{pH}$. The daily salivary volume is between 0.5 to 2 liters and it is this amount of fluid that is available to hydrate oral mucosal dosage forms. A main reason behind the selection of hydrophilic polymeric matrices as vehicles for oral transmucosal drug delivery systems is this water rich environment of the oral cavity.

\begin{abstract}
Absorption via buccal mucosa: There are two permeation pathways for passive drug transport across the oral mucosa: Para cellular and Tran cellular routes. Permeants can use these two routes simultaneously, but one route is usually preferred over the other depending on the physicochemical properties of the diffusant. Since the intercellular spaces and cytoplasm are hydrophilic in character, lipophilic compounds would have low solubilities in this environment. The cell membrane, however, is rather lipophilic in nature and hydrophilic solutes will have difficulty permeating through the cell membrane due to a low partition coefficient.
\end{abstract}


Therefore, the intercellular spaces pose as the major barrier to permeation of lipophilic compounds and the cell membrane acts as the major transport barrier for hydrophilic compounds. Since the oral epithelium is stratified, solute permeation may involve a combination of these two routes. The route that predominates, however, is generally the one that provides the least amount of hindrance to passage.

\section{Promoting buccal absorption:}

Absorption enhancers: Absorption enhancers have demonstrated their effectiveness in delivering high molecular weight compounds, such as peptides, that generally exhibit low buccal absorption rates. These may act by a number of mechanisms, such as increasing the fluidity of the cell membrane, extracting inter/intracellular lipids, altering cellular proteins or altering surface mucin. The most common absorption enhancers are azone, fatty acids, bile salts and surfactants such as sodium dodecyl sulfate. Solutions/gels of chitosan were also found to promote the transport of mannitol and fluorescent-labeled dextrans across a tissue culture model of the buccal epithelium while Glyceryl monooleates were reported to enhance peptide absorption by a co-transport mechanism ${ }^{10}$.

TABLE 1: LIST OF PERMEATION ENHANCERS ${ }^{15}$

\begin{tabular}{|c|c|c|c|}
\hline Sr. no & Permeation Enhancers & Sr. no & Permeation Enhancers \\
\hline 1 & 2, 3-Lauryl ether & XIV & Phosphatidylcholine \\
\hline II & Aprotinin & $x V$ & Polyoxyethylene \\
\hline III & Azone & $\mathrm{XVI}$ & Polysorbate 80 \\
\hline IV & Benzalkonium chloride & XVII & Polyoxyethylene \\
\hline V & Cetylpyridinium chloride & XVIII & Phosphatidylcholine \\
\hline VI & $\begin{array}{c}\text { Cetyltrimethyl ammonium } \\
\text { bromide }\end{array}$ & XIX & Sodium EDTA \\
\hline VII & Cyclodextrin & $x X$ & Sodium glycocholate \\
\hline VIII & Dextran sulfate & $X X I$ & $\begin{array}{c}\text { Sodium } \\
\text { glycodeoxycholate }\end{array}$ \\
\hline IX & Glycol & XXII & Sodium lauryl sulfate \\
\hline$x$ & Lauric acid & XXIII & Sodium salicylate \\
\hline$X I$ & Lauric acid/Propylene & XXIV & Sodium taurocholate \\
\hline XII & Lysophosphatidylcholine & $x X V$ & $\begin{array}{l}\text { Sodium } \\
\text { taurodeoxycholate }\end{array}$ \\
\hline XIII & Menthol & XXVI & Sulfoxides \\
\hline
\end{tabular}

Prodrugs: Hussain et al., delivered opioid agonists and antagonists in bitter less prodrug forms and found that the drug exhibited low bioavailability as prodrug. Nalbuphine and naloxone bitter drugs, when administered to dogs via the buccal mucosa, causes excess salivation and swallowing. As a result, the drug exhibited low bioavailability. Administration of nalbuphine and naloxone in prodrug form caused no adverse effects, with bioavailability ranging from 35 to $50 \%$ showing marked improvement over the oral bioavailability of these compounds, which is generally $5 \%$ or less ${ }^{14}$.

pH: Shojaei et al., evaluated permeability of acyclovir at $\mathrm{pH}$ ranges of 3.3 to 8.8 , and in the presence of the absorption enhancer, sodium glycocholate. The in vitro permeability of acyclovir was found to be $\mathrm{pH}$ dependent with an increase in flux and permeability coefficient at both $\mathrm{pH}$ extremes ( $\mathrm{pH} 3.3$ and 8.8), as compared to the mid-

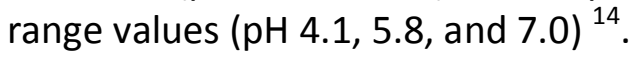

Buccal mucosa-site for drug delivery: Controlled drug delivery systems specifically designed for buccal cavity, where the drug releases in a controlled manner. The drug can be administered for local or systemic action. These systems are generally based on the polymers including bioadhesive polymers. The various dosage forms including buccal bioadhesive tablets, laminated film, hydrogels, buccal patches, chewing gums and hollow fibers have been designed to extend the time of drug release from buccal cavity.

The absorption of drug through buccal mucosa can be increased using some absorption enhancers. Different peptides including insulin can be delivered to or through buccal cavity using control drug delivery systems. Particulate systems such as microspheres and nanoparticles have also been tried for the buccal control drug delivery. Buccal control drug delivery can be achieved in 
three ways; delivery through buccal mucosa, delivery through sublingual mucosa and local delivery to mouth. Local delivery includes the systems designed mainly to deliver drugs to periodontal pocket. Bioadhesion is a major approach involved in the designing of buccal controlled drug delivery systems.

Theoretically, maximum buccal residence time can be in the order of several days. But it has been observed that usually it does not exceed several hours, possibly due to interference with drinking, eating and talking.

Dynamics: The oral mucosal absorption of drugs could be adequately described by first order rate process. Several potential barriers to oral mucosa drug absorption have been identified. These include the mucus layer, keratinized layer, intercellular lipid of epithelium, basement membrane and lamina propria. In addition, the absorptive membrane thickness, blood supply blood/lymph drainage cell renewal and enzyme content will all contribute to reducing the rate and amount of drug entering the systemic circulation. Dearden and Tomlison (1971) pointed out that salivary secretion alters the buccal absorption kinetics from drug solution by changing the concentration of drug in the mouth. They proposed a linear relationship between salivary secretion and time thus

$$
-\frac{d m}{d t}=\frac{K C}{V i V t}
$$

where ' $\mathrm{m}$ ' and ' $\mathrm{C}$ ' are the mass and concentration of drug in mouth at time ' $t$ ', $\mathrm{Vi}$, the volume of solution put into mouth cavity and ' $\mathrm{V}$ ' is salivary secretion rate. T. Suzuki et al designed a new perfusion system to study oral mucosal absorption drug using salicylic acid as a model drug in oral perfusion medium. They proposed following three compartment models.

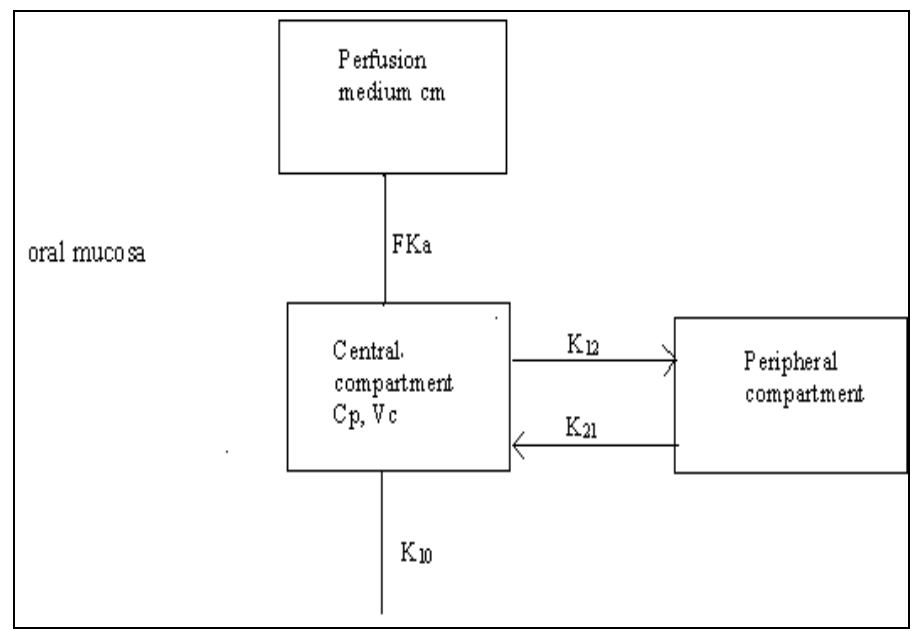

PHARMACOKINETIC MODEL FOR SALICYLIC ACID IN DOGS

$\mathrm{Cp}$, drug concentration in plasma; $\mathrm{Cm}$, drug concentration in perfusion medium; $\mathrm{Ka}$, first-order absorption rate constant; $F$, fraction of disappeared drug transferred to circulating blood; K12 and K21, first order transfer rate constants between two compartment $\mathrm{K} 10$ first order elimination rate constant: Vdc distribution volume of control compartment; Vdt distribution volume of peripheral compartment ${ }^{17,18}$.

Factors Affecting Buccal Absorption: The oral cavity is a complex environment for drug delivery, as there are many interdependent and independent factors which reduces the absorbable concentration at the site of absorption.

Membrane Factors: This involves degree of keratinization, surface area available for absorption, mucus layer of salivary pellicle, intercellular lipids of epithelium; basement membrane and lamina propria. In addition, the absorptive membrane thickness, blood supply/ lymph drainage, cell renewal and enzyme content will all contribute to reducing the rate and amount of drug entering the systemic circulation.

\section{Environmental Factors:}

- Saliva: The thin film of saliva coats throughout the lining of buccal mucosa and is called salivary pellicle or film. The thickness of salivary 
film is 310.07 to $0.10 \mathrm{~mm}$. The thickness, composition and movement of this film effects buccal absorption.

- Salivary glands: The minor salivary glands are located in epithelial or deep epithelial region of buccal mucosa. They constantly secrete mucus on surface of buccal mucosa. Although, mucus helps to retain mucoadhesive dosage forms, it is potential barrier to drug penetration

Movement of oral tissues: Buccal region of oral cavity shows less active movements. The mucoadhesive polymers are to be incorporated to keep dosage form at buccal region for long periods while withstanding tissue movements during talking and if possible during eating food or swallowing ${ }^{18,}$ $19,20,21$

Advantages of Buccal Absorption: The oral mucosa has a rich blood supply. Drugs are absorbed from the oral cavity through the oral mucosa, and transported through the deep lingual or facial vein, internal jugular vein and braciocephalic vein into the systemic circulation. Following buccal administration, the drug gains direct entry into the systemic circulation thereby bypassing the first pass effect. Contact with the digestive fluids of gastrointestinal tract is avoided which might be unsuitable for stability of many drugs like insulin or other proteins, peptides and steroids. In addition, the rate of drug absorption is not influenced by food or gastric emptying rate.

The area of buccal membrane is sufficiently large to allow a delivery system to be placed at different occasions, which may be advantageous if the drug delivery system or other excipients reversibly damage or irritate the mucosa. Additionally, there are two areas of buccal membranes per mouth, which would allow buccal drug delivery systems to be placed, alternatively on the left and right buccal membranes. There is good accessibility to the membranes that line the oral cavity which makes application painless and without discomfort, precise dosage form localization possible and facilitates ease of removal without significant associated pain and discomfort. Thus, patients can control the period of administration or terminate delivery in case of emergencies. The oral mucosal route has in the past exhibited better patient compliance than either the vaginal or rectal route of drug administration thus it would be anticipated that novel buccal dosage forms would be well accepted by patients. In addition, the route is not gender specific as is the case with vaginal route.

The oral mucosa is routinely exposed to a multitude of different foreign compounds and physical insult. So it has evolved a robust membrane that is less prone to irreversible damage by drug, dosage form or additives used therein. Thus, it may be feasible to include permeation enhancers in the formulation to increase systemic availability of the drug without observing permanent damaging effects.

Due to some therapeutic reasons oral cavity is the only ultimate route for drug delivery, for example, for those patients nil by-mouth, if either nausea or vomiting is a problem, if the patient is unconscious, in patients with an upper gastrointestinal tract disease or surgery, which affects gastrointestinal absorption or patient groups, which have difficulty in swallowing per oral medications e.g., very young and elderly. Additional advantages of the oral cavity as a site for systemic drug delivery include: sterile techniques are not required during manufacture or administration, the oral cavity contains teeth upon which drug delivery systems can be physically attached using dental adhesives, the oral mucosa is low in enzyme activity and enzymatic degradation is relatively slow, hence, from the point of drug inactivation, the oral musocal route would be preferred to the nasal or rectal routes $18,22,23,24$. 
Limitation in Buccal Absorption: The area of absorptive membrane is relatively smaller. If the effective area for absorption was dictated by the dimensions of a delivery system, this area then becomes even smaller.

- Saliva is continuously secreted into the oral cavity diluting drugs at the site of absorption resulting in low drug concentrations at the surface of the absorbing membrane. Involuntary swallowing of saliva results in a major part of dissolved or suspended released drug being removed from the site of absorption. Furthermore, there is risk that the delivery system itself would be swallowed.

- Drug characteristics may limit the use of the oral cavity as a site for drug delivery. Taste, irritancy, allerginicity and adverse properties such as discoloration or erosion of the teeth may limit the drug candidate list for this route. In addition, the drug should not adversely affect the natural microbial flora of the oral cavity.

- Conventional type of buccal drug delivery systems did not allow the patient to concurrently eat, drink or in some cases, talk.

- The permeability of the oral mucosa is not great compared to other mucosal membranes.

\section{Buccal Dosage Forms:}

\section{Buccal Dosage forms are meant to be placed between gingiva and cheek ${ }^{18,22,23,24}$ :}

Conventional Dosage Form: The conventional type of buccal dosage forms are buccal tablets, troches and lozenges, and mouth washers. Buccal tablets are small, flat, oval tablets and are intended to be held between the cheek and the teeth or in the cheek pouch (buccal tablets). Progesterone tablets can be administered this way. These tablets should be designed not to disintegrate but to slowly dissolve, typically over a 15 to 30 minutes period to provide for effective absorption. Troches and lozenges are two other types of tablets used in oral cavity where they are intended to exert a local effect in the mouth or throat. These tablet forms are commonly used to treat sore throat or to control coughing in common cold. Lozenges (pastiles or cough drops) are usually made with the drug incorporated in a flavored, hard-candy sugar base.

Lozenges may be made by compression but are usually formed by fusion or by a candy-molding process. Troches, on the other hand, are manufactured by compression as are other tablets. These two classes of tablets are designed not to disintegrate in the mouth but to dissolve or slowly erode over a period of perhaps 30 minute or less. A mouth wash is an aqueous solution, which is most often used for its deodorant, refreshing or antiseptic effect on buccal mucosa 25

Advanced Buccal Dosage Forms: The novel type buccal dosage forms include buccal adhesive tablets, patches, films, tapes, semisolids (ointments and gels) and powders

Bioadhesive buccal tablets: Bioadhesive buccal tablets are immobilized drug delivery system. They can be formulated into monolithic, partially coated or multilayered matrices. Monolithic tablets are easy to manufacture by conventional techniques and provide for the possibility of loading large amount of drug. Drug can be co-incorporated with an absorption enhancer, if required, partial coating of a monolithic tablet, which is not in contact with the mucosa. Such systems allow undirected drug release into salivary fluids.

In case of bilayered tablets, drug can be incorporated in the adhesive layer, which comes in contact with the mucosal surface. This drug containing mucoadhesive layer is then protected from the oral cavity environment by a second upper inert layer, which faces into the oral cavity. Alternatively, the drug can be incorporated into the 
upper non adhesive layer to release the drug into the oral cavity.

Retention of a mucosal drug delivery system is a requirement for the use of the oral mucosa for prolonged drug delivery. The use of cellulosic or acrylic polymers generally offers almost immediate, high adhesion performance for prolonged period of time even with high drug content. Factors such as nature of the polymer, the drug/polymer ratio and swelling kinetics influence the drug release from bioadhesive tablets ${ }^{16}$.

Patches, Tapes \& Films: Buccal patches consists of two ply laminates, with an aqueous solution of the adhesive polymer being cast onto an impermeable backing sheet, which was then cut to the required oval shape. A novel mucosal adhesive film called "Zilactin" - consisting of an alcoholic solution of hydroxy propyl cellulose and three organic acids, forms a film which applied to the oral mucosal surface which can be retained in place for at least 4 hours, even where challenged with fluids ${ }^{26}$.

Semisolid Preparations (Ointments and Gels): Bioadhesive gels or ointments have less patient acceptability than solid dosage adhesive forms, and most are used only for localized drug therapy within the oral cavity. One of the original oral mucosal-adhesive delivery systems- "orabase" consists of finely ground pectin, gelatin and sodium carboxy methyl cellulose dispersed in a poly (ethylene) and a mineral oil gel base, which can be maintained at its site of application for 15-150 minutes.

Powders: Yama moto et al., have described a hydroxypropyl cellulose and beclomethasonediproprionate containing powder that was sprayed onto the oral mucosa of rats. A significant increase in the residence time relative to an oral solution was seen, and $2.5 \%$ of beclomethasone was retained on buccal mucosa for over 4 hours ${ }^{25,26}$.

\section{Evaluation of Buccal Tablets:}

- In vitro swelling rate and bioadhesion studies

- In vitro surface $\mathrm{pH}$ studies

- In vitro drug release studies

- In vitro permeation studies

- In vitro mucoadhesion strength

- In vitro residence time

- In vivo release studies

- Stability studies in human saliva

- Ex vivo mucoadhesion time

- Ex vivo mucoadhesion force

- Ex vivo transmucosal permeation studies

\section{Experimental Methodology for Buccal Permeation} Studies: Before a buccal drug delivery system can be formulated, buccal absorption/permeation studies must be conducted to determine the feasibility of this route of administration for the candidate drug. These studies involve methods that would examine in vitro and/or in vivo buccal permeation profile and absorption kinetics of the drug.

In vitro Methods: At the present time, most of the in vitro studies examining drug transport across buccal mucosa have used buccal tissues from animal models. Animals are sacrificed immediately before the start of an experiment. Buccal mucosa with underlying connective tissue is surgically removed from the oral cavity, the connective tissue is then carefully removed and the buccal mucosal membrane is isolated. The membranes are then placed and stored in ice-cold $\left(4^{\circ} \mathrm{C}\right.$ ) buffers (usually Krebs buffer) until mounted between side-by-side diffusion cells for the in vitro permeation experiments. The most significant questions concerning the use of animal tissues as in vitro models in this manner are the viability and the integrity of the dissected tissue. How well the dissected tissue is preserved is an important issue 
which will directly affect the results and conclusion of the studies. To date, there are no standard means by which the viability or the integrity of the dissected tissue can be assessed. Dowty et al., ${ }^{28}$ studied tissue viability by using ATP levels in rabbit buccal mucosa. Using ATP levels as an indicator for tissue viability is not necessarily an accurate measure, however.

Dowty et al., reported a $50 \%$ drop in the tissue ATP concentration during the initial 6 hours of the experiment without a corresponding drop in tissue permeability. Despite certain gradual changes, the buccal tissue seems to remain viable for a rather long period of time. Therefore, a decrease in ATP levels does not assure a drop in permeability characteristics of the tissue. The most meaningful method to assess tissue viability is the actual permeation experiment itself, if the drug permeability does not change during the time course of the study under the specific experimental conditions of $\mathrm{pH}$ and temperature, then the tissue is considered viable.

Buccal cell cultures have also been suggested as useful in vitro models for buccal drug permeation and metabolism 27, 29-31. However, to utilize these culture cells for buccal drug transport, the number of differentiated cell layers and the lipid composition of the barrier layers must be well characterized and controlled. This has not yet been achieved with the buccal cell cultures used thus far.

In vivo Methods: In vivo methods were first originated by Beckett and Triggs ${ }^{32}$ with the socalled buccal absorption test. Using this method, the kinetics of drug absorption was measured. The methodology involves the swirling of a $25 \mathrm{ml}$ sample of the test solution for up to 15 minutes by human volunteers followed by the expulsion of the solution. The amount of drug remaining in the expelled volume is then determined in order to assess the amount of drug absorbed. The drawbacks of this method include salivary dilution of the drug, accidental swallowing of a portion of the sample solution, and the inability to localize the drug solution within a specific site (buccal, sublingual, or gingival) of the oral cavity. Various modifications of the buccal absorption test have been carried out ${ }^{33-36}$ correcting for salivary dilution and accidental swallowing, but these modifications also suffer from the inability of site localization. A feasible approach to achieve absorption site localization is to retain the drug on the buccal mucosa using a bioadhesive system 37-39. Pharmacokinetic parameters such as bioavailability can then be calculated from the plasma concentration vs. time profile.

Other in vivo methods include those carried out using a small perfusion chamber attached to the upper lip of anesthetized dogs 40, 41. The perfusion chamber is attached to the tissue by cyanoacrylate cement. The drug solution is circulated through the device for a predetermined period of time and sample fractions are then collected from the perfusion chamber (to determine the amount of drug remaining in the chamber) and blood samples are drawn after 0 and 30 minutes (to determine amount of drug absorbed across the mucosa).

Experimental Animal Species: Aside from the specific methodology employed to study buccal drug absorption/permeation characteristics, special attention is warranted to the choice of experimental animal species for such experiments. For in vivo investigations, many researchers have used small animals including rats $42,43,44$ and hamsters ${ }^{45,46,47}$ or permeability studies. However, such choices seriously limit the value of the data obtained since, unlike humans, most laboratory animals have an oral lining that is totally keratinized. The rat has a buccal mucosa with a very thick, keratinized surface layer. The rabbit is the only laboratory rodent that has non-keratinized mucosal lining similar to human tissue and has been 
extensively utilized in experimental studies $28,48,49$, 51,52 . The difficulty in using rabbit oral mucosa, however, is the sudden transition to keratinized tissue at the mucosal margins making it hard to isolate the desired non-keratinized region ${ }^{53}$. The oral mucosa of larger experimental animals that has been used for permeability and drug delivery studies include monkeys ${ }^{54}$, dogs ${ }^{35,40,55,56}$ and pigs $57,58,59-63$

Due to the difficulties associated with maintenance of monkeys, they are not very practical models for buccal drug delivery applications. Instead, dogs are much easier to maintain and considerably less expensive than monkeys and their buccal mucosa is nonkeratinized and has a close similarity to that of the human buccal mucosa. Pigs also have nonkeratinized buccal mucosa similar to that of human and their inexpensive handling and maintenance costs make them an equally attractive animal model for buccal drug delivery studies.

In fact, the oral mucosa of pigs resembles that of human more closely than any other animal in terms of structure and composition 64, 65 . However, for use in in vivo studies pigs are not as ideal as dogs due to their rapid growth which renders the animal handling rather difficult. Miniature breeds of pigs can be used but their high cost is a deterrent. For in vitro studies though, because of easy availability and low cost porcine tissue is more suited as compared to dog buccal tissue.

CONCLUSION: The buccal mucosa offers several advantages over controlled drug delivery for extended periods of time. The mucosa is well supplied with both vascular and lymphatic drainage and first-pass metabolism in the liver and presystemic elimination in the gastrointestinal tract are avoided. The area is well suited for a retentive device and appears to be acceptable to the patient. With the right dosage form design and formulation, the permeability and the local environment of the mucosa can be controlled and manipulated in order to accommodate drug permeation. Buccal drug delivery is a promising area for continued research with the aim of systemic delivery of orally inefficient drugs as well as a feasible and attractive alternative for non-invasive delivery of potent peptide and protein drug molecules. However, the need for safe and effective buccal permeation absorption enhancers is a crucial component for a prospective future in the area of buccal drug delivery.

\section{REFRENCES:}

1. W. (Curatolo (1987) Pharm. Res.4 271

2. N.H.F. Ho and W.I Higuchi. (1971) J.Pharm.Sci. 60,537

3. Galey, W.R., Lonsdale, H.K., and Nacht, S., The in vitro permeability of skin and buccal mucosa to selected drugs and tritiated water, J. Invest. Dermat. 1976; 67:713-717.

4. Harris, D. and Robinson, J.R., Drug delivery via the mucous membranes of the oral cavity, J. Pharm. Sci., 1992;81:1-10. Reproduced with permission of the American Pharmaceutical Association

5. Gandhi, R.B. and Robinson, J.R., Oral cavity as a site for bioadhesive drug delivery, Adv. Drug Del. Rev., 1994;13:4374.

6. Squier, C.A. and Hall, B.K., The permeability of mammalian non-keratinized oral epithelia to horseraddish peroxidase applied in vivo and in vitro, Arch. Oral Biol., 29:45-50, 1984.

7. Hill, M.W. and Squier, C.A., The permeability of oral palatal mucosa maintained in organ culture, J. Anat., 1979;128:169178.

8. P.W Wertz, and C.A Squier, Cellular and molecular basis of barrier function in oral epithelium, Crit. Rev. Ther. Drug Carr. Sys. 1991; 8:237-269.

9. Tabak, L.A., Levine, M.J., Mandel, I.D. and Ellison, S.A., Role of salivary mucins in the protection of the oral cavity, J. Oral Pathol., 1982;11:1-17.

10. Peppas, N.A., and Buri, P.A., Surface, interfacial and molecular aspects of polymer bioadhesion on soft tissues, $J$. Control. Rel., 1985; 2:257-275.

11. Rathbone, M., Drummond, B and Tucker, I., Oral cavity as a site for systemic drug delivery, Adv. Drug Del. Rev., 1994;13:1-22.

12. Gandhi, R.E., and Robinson, J.R., Bioadhesion in drug delivery, Ind. J. Pharm. Sci., 1988; 50:145-152.

13. Edgar, W.M Saliva: its secretion, composition and functions, Br. Dent. J., 1992; 172:305-312.

14. Deirdre Faye Vaughan, Pharmacokinetics of Albuterol and Butorphanol Administered Intravenously and via a Buccal Patch, A Thesis Submitted to the office of Graduate Studies 
of Texas A\&M University In Partial Fulfillment of the requirements for the Degree of Master of Science, May 2003

15. Shojaei Amir H, Buccal Mucosa as A Route for Systemic Drug Delivery, Journal of Pharmacy and Pharmaceutical Sciences, 1998; 1(1), 15-30.

16. Vyas S.P. and Khar R.K.:Controlled Drug Delivery,concepts and advances.Vallabh Prakashan,Delhi,Edition 1,2000:155195.

17. TM Pramod Kumar, HG Shivakumar and KG Desai. Oral transmucosal drug delivery systems. Indian Drug 2004 February; 41(2): 63-12.

18. Katarina Edsman and Helene Hagerstrom. Pharmaceutical applications of mucoadhesion for the non-oral routes. Journal of Pharmacy \& Pharmacology 2005; 57: 3-19.

19. Madhusudan Rao Y, Vani G, R. Rameshachary Bala. Design and evaluation of mucoadhesive drug delivery systems. Indian Drug 1998 Sept.; 35(9):

20. Bioadhesive Drug Delivery Systems. 1st Edition, Edited by James Swarbrick, Published by Marcel Dekker Inc.m New York 1999; 1-9: 541- 562.

21. Chatterjee CC. Human Physiology, 10th Edition, Published by: Medical Allied Agency, Calcutta: 1985; 427-434.

22. Tora-Tora Grabowski. Principles of Anatomy \& Physiology. 7th Edition, Edited by Gerard J. Toratora and Sandra Reynolds Grabouski, Published by Harper Collins College Publishers; 1992; 770-774.

23. Jain N.K.: Controlled and Novel Drug Delivery.CBS Publishers and Distributors,New Delhi,Edition1,1997:52-81.

24. Bhaskara Jasti DVS, Xiaoling Li and Gary C Leary. Recent advances in mucoadhesive drug delivery system. Pharmatech. 2003. www.pharmtech.com.

25. Sevdsenel, Atilla A Hincal. Drug permeation enhancement via buccal route: Possibilities and limitations. Journal of Controlled Release 2001; 72: 133-11.

26. Hung Seng Ch NG, Haesun Park, Preggy Kelly and Joseph R Robinson. Bioadhesive polymers as platforms for oral controlled drug delivery-II: Synthesis and evaluation of some swelling water-insoluble bioadhesive polymers. Journal of Pharmaceutical Science 1985 April; 74(4): 399-10.

27. Hill, M.W. and Squier, C.A., The permeability of oral palatal mucosa maintained in organ culture, J. Anat., 1979;128:169178.

28. Dowty, M.E., Knuth, K.E, Irons, B.K., and Robinson, J.R., Transport of thyrotropin releasing hormone in rabbit buccal mucosa in vitro, Pharm. Res., 1992;9:1113-1122.

29. Tavakoli-Saberi, M.R and Audus, K.L., Cultured buccal epithelium: an in vitro model derived from the hamster pouch for studying drug transport and metabolism, Pharm. Res., 1989; 6:160-162.

30. Tavakoli-Saberi, M.R., Williams, A., and Audus, K.L, Aminopeptidase activity in human buccal epithelium and primary cultures of hamster buccal epithelium, Pharm. Res., 1989; 6:S-197.

31. Leipold, H.R and Quadros, E Nicotine permeation through buccal cultures, proceed. Int. Symp. Control. Rel. Bioact. Mater. 1993; 20:242-243.
32. Beckett, A.H. and Triggs, E.J., Buccal absorption of basic drugs and its application as an in vivo model of passive drug transfer through lipid membranes, J. Pharm. Pharmacol., 1967; 19:31S-41S.

33. Schurmann, W and, Turner P., A membrane model of the human oral mucosa as derived from buccal absorption performance and physicochemical properties of the betablocking drugs atenolol and propranolol, J. Pharm. Pharmacol., 1978;30:137-147.

34. Tucker, I.G., A method to study the kinetics of oral mucosal drug absorption from solutions, J. Pharm. Pharmacol., 1988; 40:679-683.

35. Barsuhn, C.L., Olanoff, L.S., Gleason, D.D., Adkins, E.L., and N.F.H., Ho, Human buccal absorption of flubriprofen, Clin. Pharmacol. Ther. 1988; 44:225-231.

36. Gonzalez-Younes, I., J.G., D.A Wagnerand Gaines, Absorption of flurbiprofen through human buccal mucosa, $J$. Pharm. Sci., 1991; 80:820-823.

37. Benes $\mathrm{L}$, et al. Plasma melatonin (M) and sulfatoxymelatonin (aMT6s) kinetics after transmucosal administration to humans, in eds. Y. Touitou, J. Arendt, andP. Pevet, Melatonin and the pineal gland-From basic science to clinical application, Elsevier Science Publishers B.V., New York, 1993; 347-350.

38. McQuinn, R.L., Kvam D.C, Maser M.J, Miller, A.L., and Oliver, A., Sustained oral mucosal delivery in human volunteers of buprenorphine from thin non-eroding mucoadhesive polymeric disks, J. Control. Rel., 1995; 34:243-250.

39. 39. Benes $\mathrm{L}$, et al., Transmucosal, oral controlled-release, transdermal drug administration in human subjects: A crossover study with melatonin, Journal of Pharm. Sci., 86:1115-1119, 1997.

40. Veillard M.M, Longer M.A, Martens T.W, and Robinson J.R, Preliminary studies of oral mucosal delivery of peptide drugs, J. Control. Rel., 1987; 6:123-131.

41. Yamahara $\mathrm{H}$, and Lee, V.H., Drug Metabolism in the Oral Cavity, Adv. Drug Del. Rev., 1993; 12:25-39.

42. Aungst, B.J, Rogers, N.J., and Shefter E. Comparison of nasal, rectal, buccal, sublingual and intramuscular insulin efficacy and the effects of a bile salt absorption promoter, The J. Pharmacol. Exp. Ther., 1988; 244:23-27.

43. Aungst, B.J. and Rogers, N.J., Comparison of the effects of various transmucosal absorption promoters on buccal insulin delivery, Int. J. Pharm., 1989;53:227-235.

44. 44. Siegel,. I.A. and , H.P., GordonSurfactant-induced increase of permeability of rat oral mucosa to nonelectolytes in vivo, Arch. Oral Biol., 1985;30:43-47.

45. 45. Hisaichi Y S, Hong L, Nakayama T, and Kimura T,Enhanced permeability of keratinized oral-mucosa to salicylic acid with 1-dodecylacycloheptan-2-one (Azone). In vitro studies in hamster cheek pouch, Int. J. Pharm., 1989; 49:47-55.

46. Kurosaki Y, Hisaichi S, Hamada C, Nakayama T, and Kimura, T., Effects of surfactants on the absorption of salicylic acid from hamster cheek pouch as a model of keratinized oral mucosa, Int. J. Pharm., 1988; 47:13-19. 
47. Tanaka M, Yanagibashi N, Fukuda $\mathrm{H}$, and Nagai $\mathrm{T}$, Absorption of salicylic through the oral mucous membrane of hamster cheek pouch, Chem. Pharm. Bull., 1980;28:1056-1061.

48. Oh C.K, and Ritschel W.A, Biopharmaceutic aspects of buccal absorption of insulin, Meth. Find Exp. Clin. Pharmacol., 1990;12:205-212.

49. Siegel I.A, Izutsu K.T, and Watson E, Mechanisms of nonelectrolyte penetration across dog and rabbit oral mucosa in vitro, Arch. Oral Biol., 1981;26:357-361.

50. Siegel I.A., and Gordon H.P, Surfactant-induced alterations of permeability of rabbit oral mucosa in vitro, Exp. Mol. Path., 1986;44:132-137.

51. Dowty M.E, Knuth K.E, and Robinson J.R, Enzyme characterization studies on the rate-limiting barrier in rabbit buccal mucosa, Int. J. Pharm., 1992; 88:293-302.

52. Squier, C.A and Wertz, P.W. Structure and function of the oral mucosa and implications for drug delivery, in eds. M.J. Rathbone, Oral Mucosal Drug Delivery, Marcel Dekker, Inc., New York, New York, 1996; 1-26.

53. Mehta M, Kemppainen B.W, and Staffford R.G,In vitro penetration of tritium-labelled water (THO) and [3H]PbTx-3 (a red tide toxin) through monkey buccal mucosa and skin, Tox. Lett., 1991;55:185-194.

54. Nagai T, Adhesive topical drug delivery system, J. Control. Rel., 2:121-134, 1985.

55. Ishida M, Machida $Y$, Nambu $N$, and Nagai $T$, New mucosal dosage form of insulin, Chem. Pharm. Bull., 1981; 29:810816.

56. Hoogstraate A.J, Verhoef J.C, Tuk B, Pijpers A, L.A.M.G van leengoed, J.H.M Vheijden, H.E Junjinger,., and H.E Bodde, Buccal delivery of fluorescein isothiocyanate-dextran 4400 and the peptide drug buserelin with glycodeoxycholate as an absorption enhancer in pigs, J. Control. Rel., 1996; 41:77-84.

57. Hoogstraate A Senel.J,S, Cullander C, Verhoef J, Junginger H.E, and Bodde H.E, Effects of bile salts on transport rates and routes of FTIC-labelled compounds across porcine buccal epithelium in vitro, J. Control. Rel., 1996; 40:211-221.

58. Lesch C.A, Squier C.A, Cruchley A, Williams D.M, and Speight $P$, The permeability of human oral mucosa and skin to water, J. Dent. Res., 68:1345-1349, 1989.

59. Quadros E, Cassidy J, Gniecko K, and LeRoy S, Buccal and colonic absorption of CGS 16617, a novel ACE inhibitor, J. Control. Rel., 1991; 19:77-86.

60. Squier C.A, and Hall B.K, The permeability of skin and oral mucosa to water and horseradish peroxidase as related to the thickness of the permeability barrier, The J. Invest. Dermat. 1985; 84.

61. Hoogstraate A.J, Cullander C, Nagelkerke J.F, Verhoel J, Junginger H.E, and Bodde H.E, Diffusion rates and transport pathways of FITC-labelled model compounds through buccal epithelium, Proceed. Int. Symp. Control. Rel. Bioact. Mater. 1993; 20:234-235.

62. Hoogstraate A.J, Bodde H.E, Cullander C, and Junginger H.E, Diffusion rates and transport pathways of FITC-labelled model compounds through buccal epithelium, Pharm. Res., 1992;9: S-188.

63. Squier C.A, Cox P, and Wertz P.W, Lipid content and water permeability of skin and oral mucosa, The J. Invest. Dermat. 1991; 96:123-126.

64. Collins P., Laffoon J,and Squier C.A, Comparative study of porcine oral epithelium, J. Dent. Res., 1981; 60:543. 